\title{
Semántica de los términos medicina legal y medicina forense
}

\author{
Semantics of the terms legal medicine and forensic medicine
}

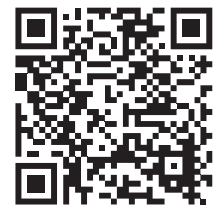

Ismael García Garduza*

\section{RESUMEN}

Objetivo: Con base en investigación bibliográfica, se analizará la importancia de la lingüística en el ámbito médico y conocerán las definiciones que se dan de la Medicina Legal y la Medicina Forense y a otros vocablos, para saber si están apegados a la semántica y, de no ser así, precisarlos para mejorar y unificar, en México, el lenguaje médico. Desarrollo del tema. Se da a conocer la definición de palabras como: diente, autopsia, necropsia y necrotomía y si esas descripciones son acordes al uso que se les da en la práctica médico-forense. Se transcriben las definiciones y competencias que se le atribuyen a la «Jurisprudencia Médica», «Medicina Legal» y «Medicina Forense», a nivel nacional e internacional y se comparan las de diferentes autores para conocer cuáles están basadas en el buen uso de la lingüística, finalizando con definiciones precisas, esperando estandarizar el buen uso del lenguaje y el razonamiento lexicográfico. Conclusión: Se establecieron definiciones y ámbitos de competencia de la Medicina Legal y la Medicina Forense, para lograr, en México, programas de enseñanza coherentes y sea real el conocimiento profesional, académico, lexicográfico, social y jurídico de las atribuciones de cada una de esas especialidades médicas.

Palabras clave: Lingüística, semántica, medicina forense, medicina legal, jurisprudencia médica.

\begin{abstract}
Objective: Based on bibliographic research, the importance of linguistics in the medical field will be analyzed and we will know the definitions that are given of Legal Medicine and Forensic Medicine and other words, to know if they are attached to semantics and, if not, specify them to improve and unify, in Mexico, the medical language. Development of the topic. The definition of words such as: tooth, autopsy, autopsy and necrotomy is disclosed, and whether these descriptions are consistent with the use given to them in forensic medical practice. The definitions and competences attributed to the «Medical Jurisprudence», «Legal Medicine» and «Forensic Medicine» are transcribed, nationally and internationally, and those of different authors are compared to know which ones are based on the good use of the linguistics, ending with precise definitions, hoping to standardize the good use of language and lexicographical reasoning. Conclusion: Definitions and areas of competence of legal medicine and forensic medicine were established, in order to achieve coherent teaching programs in Mexico and make professional, academic, lexicographic, social and legal knowledge of the attributions of each of these medical specialties are real.
\end{abstract}

Keywords: Linguistics, semantics, forensic medicine, legal medicine, medical jurisprudence.
* Especialidad en Medicina Forense cursada en el Instituto Politécnico Nacional y el Servicio Médico Forense del Distrito Federal. Perito Médico en la Procuraduría General de Justicia del Estado de México, en la Procuraduría General de la República y en la Secretaría de Salud del Gobierno del Distrito Federal. Visitador Adjunto, Coordinador de Servicios Periciales y Asesor de la Presidencia en la Comisión Nacional de los Derechos Humanos. Ha sido profesor de la cátedra de Medicina Forense en la Facultad de Derecho de la UNAM y profesor de la Cátedra de Medicina Legal en la Facultad de Medicina de la UNAM.

Correspondencia: IGG, ismaelgarciagarduza@ yahoo.com.mx Conflicto de intereses: No existen conflicto de intereses en el contenido y publicación de este artículo.

Citar como: García GI.

Semántica de los términos medicina legal y medicina forense. Rev CONAMED. 2020; 25(2): 89-94. doi: $10.35366 / 94392$

Financiamiento: Ninguno.

Recibido: 30/04/2020

Aceptado: 15/06/2020 


\section{INTRODUCCIÓN}

La lingüística, como estudio científico del lenguaje, tiene un papel crucial en la ejecución e instrucción del idioma. El lenguaje es un sistema de entidades interdependientes: fonología (estudia los patrones de sonido del lenguaje humano); morfología (analiza la palabra, sus raíces, formas y afijos, inflexiones y derivaciones); sintaxis (trata con el cómo las palabras se combinan ordenadamente en unidades más grandes para la formación de frases y oraciones); semántica (ciencia del significado de una expresión lingüística que da acceso a un determinado contenido conceptual).' Lexicografía es el arte de compilar, escribir y editar diccionarios y analizar y describir las relaciones semánticas, sintagmáticas y paradigmáticas dentro del léxico de un idioma. ${ }^{2}$

Un objetivo de la lingüística en medicina es proporcionar un análisis cuidadoso y sistémico del lenguaje y del significado que se le da a diferentes vocablos y conceptos, por ejemplo: «diente», «autopsia», «necropsia»y, en particular, a los términos «Medicina Forense»y «Medicina Legal», con los que se podrán especificar las atribuciones y utilidad de estas especialidades médicas en casos específicos, y las personas que editan diccionarios podrán utilizar esta investigación para comunicar con precisión las designaciones de cada concepto y los programas de enseñanza contendrán la temática exclusiva para cada materia.

\section{Objetivo}

Realizar una investigación bibliográfica de cómo se designan, clasifican y asignan funciones a la medicina forense y a la medicina legal en el ámbito internacional y nacional, verificar si son correctas y, de no ser así, utilizando la lingüística, alcanzar precisión en ellas y lograr que se conozcan y estandaricen esas cualidades.

\section{ERRORES COMUNES EN LA DEFINICIÓN DE CONCEPTOS}

En el ámbito jurídico, sería absurdo argumentar que la semántica y la sintaxis no son importantes, sobre todo en las áreas de Medicina Legal y Medicina Forense, ya que los dictámenes y declaraciones de peritos de esas áreas, al introducirse en un juicio, si no son expresados correctamente, pueden originar equivocaciones o imprecisiones que modifiquen el sentido del razonamiento y sean cuestionadas o impugnadas por los abogados.

Igualmente, en el área médico-forense, los vocablos «diente», «autopsia»y «necropsia» deben utilizarse con base en la semántica.

Una designación inapropiada de diente (que se define como un órgano, siendo solamente un componente del órgano de la masticación $\left.{ }^{3}\right)$, origina una clasificación médico-legal equivocada de las lesiones, afectando a una persona.

También sucede con los términos autopsia y necropsia, utilizados indistintamente para definir la disección de cadáveres, a pesar de que el vocablo autopsia proviene de «autos» $\mathrm{y}$ «opsis» que significan «sí mismo» y «ver», respectivamente, que en realidad es la auto-observación de algo sin tocarlo; científicos forenses en Grecia usan este término para referirse al cuidadoso examen de la escena de la muerte sin destruir evidencia. Por otro lado, la palabra griega necropsia proviene de «necros» y "opsis»' que significan «muerto» $y$ «ver», respectivamente, literalmente un «mira el cadáver»; es un examen externo cuidadoso del cuerpo intacto (aún no disecado) para deducir o confirmar, si es posible, la causa de la muerte.,5

Esos vocablos no expresan lo que realmente se efectúa en un estudio postmortem médico-forense, donde se realiza la disección del cuerpo humano abriendo las cavidades y examinando los órganos, por lo que el vocablo más correcto sería necrotomía que, de acuerdo con el diccionario MerriamWebster, etimológicamente procede del prefijo «necro» del griego «veкpog» (nekros) cadáver, y del

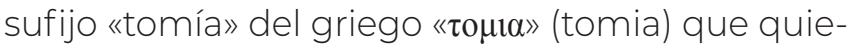
re decir corte o incisión (disección de cadáveres). En griego moderno esta es la palabra usada para describir la disección del cuerpo., ${ }^{4,5}$

Lo mismo sucede al definir a la medicina forense e igualarla a la medicina legal determinando, equivocadamente, su cometido.

Ratificando lo precedente, Putri Dianita ${ }^{6}$ expresa: «Dependiendo de dónde se practica, la medicina forense también se conoce como medicina legal, jurisprudencia médica y práctica médico-legal. La incertidumbre sobre cómo debe llamarse refleja la falta de uniformidad sobre qué es lo que comprende su práctica. El término Medicina Forense se emplea 
en los Países Bajos, Bélgica, Alemania, Francia, Suecia, Noruega, Egipto, Arabia Saudita, Turquía, Irán, Bangladés, Japón, China, Indonesia y Australia. En otros países el término Medicina Legal se usa en lugar de Medicina Forense. Como una complicación taxonómica adicional, las opiniones varían en cuanto a si la medicina forense y la medicina legal son sinónimos o si son dos entidades separadas».

El problema en establecer una semejanza de los conceptos abarca incluso diccionarios. «El proceso de consulta del diccionario es la primera etapa del aprendizaje léxico, que introduce una relativa libertad de conocimiento: el conocimiento pasivo, que es la comprensión (definición lexicográfica) y el conocimiento activo, uso adecuado de la unidad en diversos contextos lingüísticos. El Diccionario Médico para Profesiones de la Salud y Enfermería proporciona una entrada para «Medicina Forense» con dos breves definiciones que no son suficientes para definir el área de estudio; en cambio, especifica que «Medicina Legal» es sinónimo de «Medicina Forense». También, el diccionario Merriam Webster proporciona una sola entrada para «Medicina Forense» con una definición que especifica su sinonimia con «Medicina Legal». Para un no especialista, la información proporcionada por los diccionarios puede parecer confusa. En un estudio se afirma que las consecuencias de la confusión pueden ser catastróficas en consecuencia, es importante que compartamos el mismo vocabulario»?

\section{DEFINICIÓN DE MEDICINA FORENSE Y MEDICINA LEGAL O JURISPRUDENCIA MÉDICA}

Semánticamente la palabra «forense» deriva del latín «forensis» que significa «foro», lugar de reunión donde las personas con responsabilidad pública discutieron los asuntos cívicos y legales; así, la medicina forense se ocupa de la aplicación del conocimiento médico en la administración de la ley y la justicia, ${ }^{7}$ diferente a la medicina legal, que semánticamente significaría: «la medicina que está establecida por la ley o está conforme con ella», ${ }^{8}$ por tanto, es el estudio de las leyes, normas oficiales, guías prácticas de clínica, tesis, jurisprudencias y principios éticos que regulan la práctica médica.

Bardale ${ }^{9}$ refiere: «La Medicina Forense y la Jurisprudencia Médica no son términos sinónimos.
La medicina forense se ocupa de la aplicación de los conocimientos médicos en la administración de la ley y la justicia. El término Jurisprudencia médica (juris = ley, prudentia = conocimiento) trata el aspecto legal de la práctica médica. Esta rama se ocupa de las responsabilidades legales del médico mientras practica la medicina».

Guarnaluses NL ${ }^{10}$ afirma: "Derecho médico, aquella parte de la medicina legal que trata sobre la normación jurídica del ejercicio profesional de la medicina. Es término utilizado en ocasiones para sustituir al de medicina legal. Pudiera admitirse como designación sinónima de la jurisprudencia médica, también utilizada a veces con sentido más lato, como medicina legal».

Mukheriee JB" menciona: «La práctica de la medicina también se ocupa de su aspecto médicolegal, relativo a: (a) relación médico-paciente; (b) relación médico-médico; (c) relación médico-estado, deberes de un médico. La jurisprudencia médica connota el aspecto legal de la práctica médica».

Welke Barbara Y:12 «James C. Mohr, en su libro, trata dos definiciones distintas de Jurisprudencia Médica: (1) todas las interacciones entre aquellos con pericia médica y la autoridad legal y; (2) la Jurisprudencia Médica o Medicina Legal es la rama de la ciencia y la medicina que involucra a los médicos y la ley».

Estos autores permiten conocer que, por su definición y aplicación, el término «Jurisprudencia Médica» es sinónimo de Medicina Legal y a su vez, diferente a la Medicina Forense.

\section{DEFINICIONES IMPRECISAS DE MEDICINA LEGAL}

En México, Martínez Murillo13 refiere: «Medicina Legal, es el conjunto de conocimientos médicos, que tienen por objeto auxiliar a las autoridades judiciales a resolver problemas del orden penal, civil y laboral. Algunos cated ráticos consideran impropio el nombre de Medicina Legal, piensan que sería más correcto llamarle: «Medicina Forense», «Biología Jurídica», «Antropología Médica», «Jurisprudencia Médica», «Medicina Judicial», etcétera. Nosotros la seguiremos Ilamando Medicina Legal, porque con este nombre es universalmente conocida...».

En Bolivia, Nuñez de Arco $^{14}$ insiste en las distintas denominaciones: «Medicina Forense»; «Me- 
dicina Legal»; y, con una connotación más amplia, «medicina criminológica». También hay la denominación de Medicina Judicial o Jurisprudencia Médica, que de alguna manera sirve para delimitarla. El término en general se usa de forma indiscriminada y es aceptada en todas sus acepciones.

Estos dos autores, a pesar de existir otras designaciones que se hacen de la medicina legal, que denotan ambigüedad, no cuestionan si esos nombres son correctos y, sin emitir un razonamiento que las justifique, las aceptan.

\section{DEFINICIONES Y FUNCIONES DE LA MEDICINA FORENSE Y DE LA MEDICINA LEGAL ACORDES A LA LINGÜÍSTICA}

\section{Medicina Forense}

1. MA. Duque: 15 «Tradicionalmente las definiciones de medicina forense o de ciencias forenses implican que tienen como campo de acción principal la investigación de casos criminales».

2. Magdy Abdel: ${ }^{16}$ "Ciencia forense abarca un grupo creciente de subespecialidades en ciencia y medicina, las cuales transmiten métodos fundamentales, científicamente válidos y legalmente admisibles, para la presentación de pruebas en los tribunales de justicia».

3. Omo-Aghoja Lawrence: $:^{17}$ "La medicina forense es un campo que involucra la recolección y análisis de evidencia médica (muestras) para producir información objetiva para su uso en el sistema legal».

4. Theeb Alkahtani:18 «La Medicina Forense es una rama de la medicina que se especializa en la recolección y presentación de evidencia médica en disputas legales. La evidencia se puede obtener tanto de sujetos vivos como difuntos dependiendo de lo que intenta establecerse».

5. En México, el Dr. Alva Rodríguez: "19 «Es funcional definir a la Medicina Forense como la disciplina que aplica los conocimientos médicos en auxilio de la procuración y de la impartición de la justicia».

6. También en México, Fernández Pérez:20 «Medicina Forense es una disciplina de aplicación de conocimientos científicos, de índole fundamentalmente médica, para la resolución de problemas biológicos humanos que están en relación con el Derecho».

\section{Medicina Legal}

1. Lizaraso:21 "La medicina va adquiriendo un mayor perfeccionamiento técnico, para ofrecer grandes posibilidades diagnósticas y asistenciales, con lo cual surge una responsabilidad profesional más rigurosa. Aquí surge la Medicina Legal, como ciencia, que nos muestra un panorama amplio entre el trabajo asistencial del médico y su regulación normativa jurídica».

2. Suescún Vargas y colaboradores:22 «la Medicina Legal se define como la rama de la medicina que estudia los aspectos legales de la atención sanitaria».

3. Roy G Beran: ${ }^{23}$ «La medicina legal tiene mayor injerencia en el ámbito del cuidado de la salud de los pacientes. Además, las áreas que son del dominio de la medicina legal, es la aplicación de la legislación a la práctica de la medicina».

4. Aguilar Sierra:24 «Medicina Legal es un área multidisciplinaria que interactúa con muchas áreas de la medicina y es un arma que nos permite en algún momento salir bien librados de un proceso legal».

5. Hernández Sosa:25 «La medicina tiene como principal objetivo curar las enfermedades, se comprende que independientemente del propósito médico-clínico de esta ciencia, hay nuevas inquietudes y necesidades que exigen a la sociedad médica la formulación de leyes y normas para su correcta aplicación. Este conjunto de códigos y cuestiones médico-legales conforman la Medicina Legal».

6. Garay Bravo: ${ }^{26}$ "La asignatura de Medicina Legal, forma parte del campo disciplinar de Ciencias Sociales y Humanidades. Su carácter particular, por observar aspectos jurídicos en salud, la convierten en una herramienta indispensable para el ejercicio de la profesión de enfermería en el marco legal».

7. Schultz ${ }^{27}$ afirma: «La ley de la Medicina, enfatiza el aspecto de la medicina legal que se relaciona a la práctica de la medicina. Constituye la mayor parte del contenido de lo que se enseña en las facultades de derecho y en las facultades de medicina como jurisprudencia médica. Podría ayudar a centrar la atención en las diferencias entre estos dos aspectos, si el término jurisprudencia médica estuviera limitado a la ley de la 
medicina y el término medicina forense a la medicina de la ley. Medicina forense, en sentido estricto, debe ser enseñada por los médicos a los estudiantes de derecho y medicina con la mayor atención en la escuela de medicina, ya que es la ciencia médica que debe aplicarse a las necesidades de la justicia».

Estos autores definen claramente la Medicina Legal y la Medicina Forense, resaltando la indiscutible diferencia en sus ámbitos de competencia.

Por lo que se puede establecer finalmente que: Medicina Legal o Jurisprudencia Médica es el conocimiento de la ley que delimita el ejercicio ético y legal de la medicina, para la toma correcta de decisiones en el ejercicio médico-clínico; mientras que la Medicina Forense es el conocimiento médico aplicado en la investigación de delitos para la procuración y administración de justicia. ${ }^{28}$

Por ello, en la Universidad Nacional Autónoma de México, en la Carrera de Derecho se imparte la cátedra de Medicina Forense y en la Carrera de Medicina la asignatura de Medicina Legal.

Sin embargo, en la misma universidad, a la cátedra de Medicina Legal se le asignan 10-12 clases, en el cuarto año de la carrera de Medicina, de alrededor de dos a tres horas de duración cada una, tiempo insuficiente para la importancia de la materia en el desempeño profesional de los futuros médicos.

\section{DISCUSIÓN}

\section{¿Es la lingüística realmente importante para un médico?}

Tradicionalmente, la corrección del lenguaje médico ha sido uno de los parámetros para medir la calidad de los textos científicos. El lenguaje médico, como todo lenguaje científico, debe caracterizarse por su precisión y rigor, es decir, debe tener claramente definido el significado y connotación de todas las denominaciones y palabras que utiliza para evitar cualquier confusión y lograr una comunicación universal. ${ }^{29}$

Por esa falta de rigor en el lenguaje médico, el nombre de las especialidades médicas «Medicina Forense»y «Medicina Legal» ha creado incorrecciones en México sobre su significado y funciones, principalmente por la poca o nula importancia que se le da a la semántica, originando que esos conceptos se consideren sinónimos aún en los diccionariosy, en consecuencia, se enseñen y apliquen de manera equivocada ocasionando intrusismo profesional. El término «Jurisprudencia Médica», que se utiliza en otros países también, es utilizado por algunos autores mexicanos de manera defectuosa puesto que se le asemeja con la medicina forense. Estas ambigüedades provocan confusión entre los médicos y otros profesionales, en particular los abogados, que frecuentemente interactúan con la profesión médica, impidiéndoles conocer el ámbito de competencia de cada especialidad y su utilidad en casos específicos.

Por ende, resulta conveniente conocer la lingüística, sus componentes y su interacción con el ámbito médico, para que los términos y vocablos sean aplicados correctamente acorde a nuestro lenguaje y cultura. Con ello se logrará el objetivo importante de que todos los profesionales, instituciones académicas, empresas que se dedican a compilar, escribir y editar diccionarios y la sociedad compartan un vocabulario común y se eviten confusiones.

En este artículo, se ha sustentado suficientemente la diferencia semántica y actividades de las especialidades médicas: Medicina Forense y Medicina Legal. Mientras la medicina forense se relaciona con el derecho penal, los conocimientos que esta especialidad médica aporta son muy útiles para resolver casos específicos relacionados con delitos como: homicidio, lesiones, tortura, violación, accidentes automovilísticos, así como para determinar estado de ebriedad, intoxicación aguda por drogas y farmacodependencia, etcétera.

En cuanto a la medicina legal, debido a la trascendencia del acto médico en la salud y la vida de las personas, debe ser llevado a cabo acorde con la Ética y la Ley; por todo ello, la formación en el conocimiento de esas áreas, que se debe impartir en la materia de Medicina Legal, es de vital importancia para el correcto ejercicio de la profesión médica. Sin embargo, es sorprendente qué pocas horas lectivas se dedican a esta disciplina en el programa de la carrera de medicina de la UNAM.

\section{CONCLUSIÓN}

El problema en la definición de Medicina Forense y Medicina Legal es universal, esta situación no 
justifica que en México esos conceptos sean definidos de manera equivocada y a las especialidades médicas que llevan ese nombre se les asignen funciones equivocadas como en otros países, por consiguiente, si se quiere que esos defectos se corrijan en nuestro país, es necesario estandarizar semántica y lexicográficamente esos términos, con lo que se logrará que sea real el conocimiento profesional, académico, lexicográfico, social y jurídico de sus atribuciones.

\section{BiblografíA}

1. Syarif H. Linguistics and the english language instruction. Lingua Didaktika. 2016; 10 (1): 50-59.

2. Bergenholtz H. What is lexicography? Lexikos. 2012; 22: 31-42.

3. García Gl. Medicina forense. Solución a dos temas controvertidos: estado de ebriedad y lesiones dentarias. Editorial Porrúa, México, 2017. p. 62.

4. Thali M. Autopsy, necropsy, and necrotomy. Am J Forensic Med Pathol. 2012; 33 (2): e6.

5. Manning C. Points: college and non-college. Br Med J (Clin Res Ed). 1983; 287: 840.

6. Dianita IP, Freeman MD, Herkutanto, Zeegers MP. A review of the diversity in taxonomy, definitions, scope, and roles in forensic medicine: implications for evidence-based practice. Forensic Sci Med Pathol. 2018; 14: 460-468.

7. Coancá M. Strategy for enhancing skills of English for forensic medicine. Modern Journal of Language Teaching Methods (MJLTM). 2018; 8 (3): 431-440.

8. Diccionario de uso del español de América y España. Spes Editorial, S.L. Edición. Barcelona, 2002.

9. Bardale R. Principles of forensic medicine and toxicology. jaypee brothers medical publishers. Nueva Delhi, India, 2011. p. 3.

10. Berenguer GN, Arroyo TJ, Berenguer GM. Algunos preceptos y disposiciones de la teoría general del derecho [Artículo en Línea] MEDISAN 2009; 13 (1). Disponible en: http://bvs.sld.cu/revistas/san/vol13_1_09/san09109.htm.

11. Mukheriee JB. Legal aspect of medical practice. J Indian Med Assoc. 1999; 97 (11): 461-465.

12. Welke BY. Mohr James C, Doctors and the Law: Medical Jurisprudence in Nineteenth- Century America, New York:
Oxford University Press, 1993. Law and History Review, 1996; 14 (1): 185-187. doi: 10.2307/827638.

13. Martínez MS. Medicina legal. Méndez Editores. 18a. ed. México, 2012. pp. 1-2.

14. Nuñez AJ. Medicina legal y criminalística. Capítulo II La Autopsia. 3a. Ed. CTZ. Sucre, Bolivia, 2005. pp. 19-20.

15. Duque MA. El papel de la medicina forense en el contexto del trabajo humanitario. Cuad Med Forense. 2010, 16 (1-2): 37-42.

16. Kharoshah MA, Kamal ZM, Galeb SS, Reheem MA, Ahmed EE. Origin and development of forensic medicine in Egypt. J Forensic Leg Med. 2011; 18: 10-13.

17. Omo-Aghoja L. Forensic medicine: the story, clinical nexus and challenges of the practice in Nigeria. Int J of Forensic Med Invest. 2016; 2 (1): 1-4.

18. Alkahtani T, Aljerian K, Golding B, Alqahtani S. Forensic science in the context of islamic law: a review. Journal of Forensic and Legal Medicine. 2015: 34; 179-181.

19. Alva RM. Compendio de medicina forense. Méndez Editores, 5. ed., México, 2014. p. 4.

20. Fernández PR. Elementos básicos de medicina forense. Secretaría de Gobernación. México, 1976. p. 6.

21. Lizaraso CF, Pacheco CJ. Medicina Legal: ciencia útil en la prevención de denuncias de nuestros actos médicos. Horiz. Med. 2017; 17 (3): 4-5.

22. Suescún VJ, Pérez SR, Roger A, Rueda DA, Rodríguez IE. Historia de la medicina legal. Med UIS. 2009; 22 (1):83-90.

23. Beran RC. What is legal medicine - Are legal and forensic medicine the same? J Forensic Leg Med. 2010; 17: 137-139.

24. Aguilar-Sierra LE. Introducción a la medicina legal. Rev Mex Anest. 2004; 27 (1): 178-181.

25. Hernández SM, Añorga MJ, Sosa ST. Evolución histórica de la Medicina Legal y las actuaciones medicolegales en Cuba. Panorama. Cuba y Salud. 2018; 13 (3): 97-102.

26. Garay BI, García DM. Programa de la asignatura de Medicina Legal de Enfermería, de la Secretaría de Educación del Estado de México. 2010. cbt2chimalhuacan. edu.mx

27. Schultz OT. Role of medical science in the administration of criminal justice. Journal of Criminal Law and Criminology. 1933. 23 (5): 736-769.

28. García Gl. Medicina legal. Mala práctica médica y juicio oral. Editorial Porrúa, México, 2019. p. 12.

29. Benavent RA, Iscla AA. Problemas del lenguaje médico actual Extranjerismos y falsos amigos. Papeles Médicos 2001; 10 (3):144-149. 